\title{
Two-Way Impacts Between Macrophages on Vascular Endothelium and Characteristics of TCM Syndromes in Dyslipidemic Mice with the Phlegm- Dampness Retention syndrome and the Spleen and Kidney Yang Deficiency syndrome Using RNA-Seq
}

Jing Chen

The Third Affiliated Hospital, Beijing University of Chinese Medicine https://orcid.org/0000-0003-4390-8468

Chao Ye

Beijing University of Chinese Medicine Affiliated Dongzhimen Hospital

\section{Zheng Yang}

Beijing University of Chinese Medicine School of Traditional Chinese Medicine

Tieshan Wang

Beijing University of Chinese Medicine

Bing Xu

CTRC: China Tibetology Research Center

\section{Pengyang Li}

Beijing University of Chinese Medicine Affiliated Dongzhimen Hospital

Shan Zhang

Beijing University of Chinese Medicine School of Traditional Chinese Medicine

Xiaolin Xue ( $\nabla$ bjxuexiaol@163.com)

Research

Keywords: Macrophages, Traditional Chinese Medicine, Dyslipidemia, Phlegm-Dampness Retention syndrome, Spleen and Kidney Yang Deficiency syndrome, RNA-Seq

Posted Date: March 4th, 2021

DOI: https://doi.org/10.21203/rs.3.rs-259192/v1

License: (c) (i) This work is licensed under a Creative Commons Attribution 4.0 International License. Read Full License 


\section{Abstract}

Background: 'Treating the same disease with different methods' is a Traditional Chinese Medicine (TCM) therapeutic concept. That means although patients are diagnosed with the same disease, they may have different syndromes that require distinct drug administrations. This study aimed to identify the differentially expressed genes and related biological processes in dyslipidemia with the Phlegm-Dampness Retention (PDR) syndrome and the Spleen and Kidney Yang Deficiency (SKYD) syndrome using transcriptomic analysis.

Methods: Ten ApoE knockout (ApoE-/-) mice were used for the establishment of dyslipidemic disease-syndrome models via multifactor-hybrid modeling, with 5 in the the PDR group and 5 in the SKYD group. Five C57BL/6J mice were employed as normal controls (NC) group. Test model quality. Aortic endothelial macrophages in mice were screened using flow cytometry. Transcriptomic analysis was performed for macrophages using RNA-Seq.

Results: $₫$ The quality assessment of the disease-syndrome model showed that TG, TC, and LDL-C levels significantly increased in the PDR and SKYD groups versus the NC group $(P<0.05)$. Combined with HE staining of aorta, the disease model was successfully established. $\otimes T h e$ quality assessment of the syndrome models showed that mice in the PDR group presented with typical manifestations of the PDR syndrome, and mice in the SKYD group

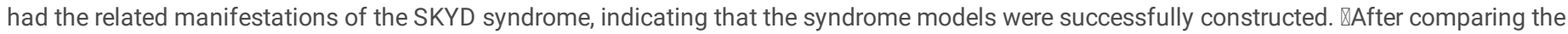
differentially expressed gene (DEG) expressions in macrophages in dyslipidemia mice with different syndromes, 4142 genes were identified with statistical significance $(P<0.05)$. The Gene Ontology $(G 0)$ analysis for the DEGs showed that biological process of difference between PDR group and SKYD group include both adverse and protective processes were included.

Conclusion: The DEGs between the PDR syndrome and the SKYD syndrome indicate different biological mechanisms between the onset of the two syndromes. They have distinctive biological processes, including adverse and protective processes, corresponding to the invasion of pathogenic factors into the body and the fight of healthy qi against pathogenic factors, respectively, in the TCM theory. Our results have demonstrated the biological evidence behind 'treating the same disease with different treatments' in TCM.

\section{Background}

As the life quality increases and dietary pattern changes, multiple factors give rise to the growing morbidity of dyslipidemia. An epidemiological study shows that dyslipidemia closely links to cardiovascular and cerebrovascular diseases, including coronary atherosclerotic heart disease and cerebral infarction [[1]]. It is estimated that the overall morbidity of dyslipidemia in Chinese adults reaches $40.40 \%$ and continues to rise [[2]]. Dyslipidemia is characterized by abnormalities in the quantity and quality of lipids in the plasma, including a lower high-density lipoprotein cholesterol (HDL-C) level and higher triglyceride (TG), total cholesterol (TC), and low-density lipoprotein cholesterol (LDL-C) levels [[3]]. Lipid and cholesterol accumulation in the vascular wall may lead to endothelial dysfunction [[4]]. Dyslipidemia is an independent and changeable risk factor shortening the onset time of atherosclerosis [[5]]. The 2013 American College Foundation of Cardiology and American Heart Association (ACCF/AHA) guideline on the management of blood lipid in atherosclerotic cardiovascular disease (ASCVD) recommended that management for dyslipidemia is the key to control risk factors of ischemic cardiovascular events [[6]].

Numerous clinical studies and laboratory experiments have ascertained the satisfactory efficacy of TCM in dyslipidemia, enriching the therapies for the disease [[7]]. A sound effect of TCM lies in the accurate discrimination of TCM syndromes of dyslipidemia, which is the basis of TCM diagnosis and treatment. The differentiation of syndromes refers to collecting clinical information (TCM symptoms) of patients via integrated TCM diagnostic methods, including looking, listening, questioning, and feeling the pulse, and analyzing and summarizing the etiological factors and pathogenesis according to the TCM thinking mode.

As patients present with different 'TCM symptoms', dyslipidemic patients may exhibit various syndromes that can be considered different TCM subtypes of dyslipidemia. The SKYD syndrome and PDR syndrome are common in dyslipidemic patients. The vascular endothelium is accountable for delivering nutrients in a dynamic way [[8]]. Dyslipidemia acts as a risk factor of cardiovascular disease probably via promoting endothelium dysfunction, a prerequisite for the occurrence of atherosclerotic manifestations [[9]]. A critical mechanism of endothelium dysfunction is oxidative stress. Excessive nitric oxide (NO) binding with hyperoxides can form peroxynitrite anion (ONOO-). ONOO- triggers oxidative stress of vascular endothelium and the endothelial injury through its oxidative effect (nitrification) on proteins, exacerbating the endothelial injury. So the severity of endothelial injury can be indicated by ONOO- levels. Our team has been studying the integration of TCM differentiation and western medicine diagnosis and investigating endothelial injury differences, as the characteristics, between the SKYD syndrome and the PDR syndrome in dyslipidemia. Our previous study suggested that dyslipidemic patients with the SKYD syndrome and the PDR syndrome exhibited different serum ONOO- concentrations, reflecting the difference in TCM syndromes in a certain sense [[10]]. It is speculated that different syndromes of dyslipidemia may correspond to different degrees of endothelial injury.

In dyslipidemia, macrophages play a pivotal role in the process of endothelial injury. Concerning the critical effect of macrophages and based on our previous study series, this work focused on aortic endothelial macrophages and explored the characteristics of the endothelial injury between the SKYD syndrome and the PDR syndrome in dyslipidemia.

Macrophages consist of two subtypes, M1 and M2 macrophages [[11]]. LDH stimulates the expressions of adhesion molecules and chemokines, thereby differentiating monocytes into macrophages through various pathways. Macrophages differentiate through different pathways. Of the two subtypes, M1 macrophages can release inflammatory factors that facilitate the progression of inflammation and further impairing vascular endothelial cells [[12]]. M2

Page 2/24 
macrophages release anti-inflammatory factors that involve in angiogenesis and tissue growth and delay the progression of inflammation [[13]]. A study showed that macrophages are indispensable in the formation of atherosclerosis [[14]]. The two subtypes exhibit antagonistic characteristics,

corresponding to 'healthy $q i$ (the ability to combat evils and preventing disease)' and 'evil qi (pathogenetic factors or factors damaging healthy $q i)$ ' in the TCM theory. The interactions between healthy qi and evil qi are characterized by 'healthy energy-evil struggles, mutually opposing and constraining, and the rule of waxing and waning' in the occurrence and development of diseases and syndromes.

Disease-syndrome combination is a significant mode for TCM diagnosis and treatment in the clinic. The in-depth investigation of diseases and syndromes calls for biological studies using disease-syndrome animal models in agreement with the traits of TCM theories to understand the underlying mechanisms. The characteristics of these models, such as rigorous control, high repeatability and success rate, make it easier to control the research cycle, repetitively perform experiments, collect more data, and comprehensively analyze the data. So the establishment of an appropriate diseasesyndrome animal model is capturing more attention.

An important way for TCM syndrome research is investigating biological mechanisms and the characteristics of syndromes using animal models. So we developed a disease-syndrome animal model in this study for analysis. The quality of a disease-syndrome animal model can affect the accuracy and credibility of a study. We employed multifactor-hybrid modeling and established dyslipidemic mouse models with the SKYD syndrome and the PDR syndrome based on the model used in our previous study so that the quality of the animal models can be ensured as much as possible. The disease model replicated the core physiopathologic process during the occurrence and development of a disease. The syndrome models simulated the core etiology and pathology during the onset of a syndrome.

Transcriptomics is a technique used to study gene transcription and regulation related to TCM syndromes and is conducive to in-depth biological research for a better understanding of the pathogenesis of TCM syndromes, which is worthy of more prevalent application in TCM syndrome research [[15]]. Currently, studies about the characteristics of the SKYD syndrome and the PDR syndrome of dyslipidemia are rarely reported. Our study focused on the two-way impacts between the severity of the endothelial injury and macrophages and analyzed the differences in biological processes and signaling pathways between different TCM syndromes (subtypes) of dyslipidemia using transcriptomic techniques. This study provided a reference for in-depth mechanical research of TCM syndromes and studies of targets of TCM drugs.

\section{Materials And Methods}

\subsection{Experimental animals}

Ten ApoE knockout mice, male, 6 weeks old, body mass about $20 \pm 5 \mathrm{~g}$. Five C57BL/6J mice of the same strain, male, 6 weeks old, body mass about $20 \pm 5 \mathrm{~g}$. All animals were raised in Beijing Changyang Xishan Farm. Rearing environment: room temperature $21-25^{\circ} \mathrm{C}$, humidity $50 \%-70 \%, 12 \mathrm{~h}$ alternating shade. The ethics of this study was approved by the animal ethics review committee of the Institute of Basic Theories of Chinese medicine, Chinese Academy of Chinese Medical Sciences, approval no. 201908006 (Beijing, China).

\subsection{Experimental main reagents and mold-making feed}

Main reagents for the experiment: LDL Cholesterol Test Kit (Nanjing Jiancheng Bioengineering Institute Co., Ltd.『; Total Cholesterol Lipoprotein Test Kit (Nanjing Jiancheng Bioengineering Institute Co., Ltd.); HDL Cholesterol Test Kit (Nanjing Jiancheng Bioengineering Institute Co., Ltd.); Triglyceride Test Kit (Nanjing Jiancheng Bioengineering Institute Co., Ltd.); FITC Anti-Mouse F4/80 Antigen (BM8.1) (Tonbo Biosciences Co., Ltd); PE Anti-Human/Mouse CD11b (M1/70) (Tonbo Biosciences Co., Ltd); Hematoxylin-eosin staining solution (Aijia Biotechnology Co., Ltd).

Experimental modeling feed: The dyslipidemic feed formula consisted of $63.6 \%$ basal feed $+15 \%$ lard $+20 \%$ sucrose $+1.2 \%$ cholesterol $+0.2 \%$ sodium cholate, provided by Beijing Keaoxieli Feed Co.

\subsection{Modeling and evaluation methods of animal models of diseases and syndromes}

Modeling methods: (1) the PDR group: 5 ApoE knockout mice were randomly selected and fed a high-fat diet for 4 weeks from weeks 1 to 4 ; (2) the SKYD group: 5 ApoE knockout mice were randomly selected and given $0.1 \%$ propylthiouracil by gavage at a dose of $10 \mathrm{mg} /(\mathrm{kg} \cdot \mathrm{d}) \mathrm{during}$ weeks $1-4$, and high-fat chow for 2 weeks during weeks 5-6; (3) the NC group: 5 C57BL/6J mice were given normal chow for modeling for a total of 4 weeks from weeks $1-4$.

Model evaluation methods: (1) evaluation of model quality of dyslipidemia by means of serum lipid index testing and aortic pathology staining; (2) evaluation of model quality of the PDR group and the SKYD group by observing the characteristic manifestations of the syndromes.

\subsection{Sampling and macrophage screening methods}

Mice were fasted without water for $18 \mathrm{~h}$ before sampling and given anesthesia for execution; (1) animal serum: animals in the PDR group, the SKYD group and the NC group, after taking whole blood, placed in conventional serum tubes, 3000 rpm for 10 minutes, separated serum, divided and immediately stored in -80 degrees Celsius refrigerator; (2) Animal tissues: aortic tissues were taken from animals in the PDR group and the SKYD group; (3) Macrophages: aorta of animals from the PDR group and the SKYD group were isolated and removed intact, digested by adding trypsin, sieved and ground, digestion was terminated, centrifuged, supernatant was decanted, resuspended by adding PBS, centrifuged again, supernatant was decanted, 
and labeled with FITC Anti-Mouse F4/80 Antigen (BM8.1) and PE Anti-Human/ Mouse CD11b (M1/70), blown and resuspended, and stored away from light. After labeling macrophages, macrophages were screened by fluorescence-activated cell sorting (FACS) using the MoFlo XDP Ultra-Fast Flow Cell Sorting System (Beckman Coulter Co., Ltd).

\subsection{Measurement of indicators}

1.5.1 General observation of the model: observing and recording mental status, body hair glossiness, movement, feces, etc.

1.5.2 Lipid index testing: serum testing of triglyceride, total cholesterol lipoprotein, high density lipoprotein cholesterol and low density lipoprotein cholesterol levels in each group.

1.5.3 Aortic histopathology: Specimens were fixed in $10 \%$ neutral formalin at room temperature for 24 hours. The specimens were fixed for 48 hours at room temperature using freshly prepared $4 \%$ formaldehyde, followed by paraffin embedding and sectioning. Specimens were cut into 4 -mm-thick sections and subjected to histological hematoxylin and eosin staining to observe the morphological features of the vascular endothelium.

After staining, the slices were photographed and examined using an optical microscope (AE41; Motic) equipped with a digital scanner (Pannoramic MIDI; 3DHISTECH) to record images of the stained slices.

\subsubsection{Macrophage transcriptomic sequencing and data analysis}

Total RNAs of macrophages were extracted in accordance with the manual of TRIzolß(Life Technologies, Inc., Gaithersburg, MD). Preparation of library and sequencing of transcriptome were carried out using Illumina HiSeq X Ten (Novogene Bioinformatics Technology Co., Ltd., Beijing,China). The mapping of 100-bp paired-end reads to genes was undertaken using HTSeq v0.6.0 software, while fragments per kilobase of transcript per million fragments mapped (FPKM) were also analyzed. Raw reads from RNA-seq libraries were trimmed to remove the adaptor sequence and the reads with adaptor contaminants and low-quality reads (the mass value Q-score $<5$ of the base number accounts for more than $50 \%$ ) and reads from $\mathrm{N}$ ( $\mathrm{N}$ indicates that the base information that cannot be determined) which is $>10 \%$. After filtering, reference genome and gene model annotation files were downloaded from a genome website browser (NCBI/UCSC/Ensembl). Indexes of the reference genome were built using Bowtie v2.0.6 and paired-end clean reads were aligned to the reference genome using TopHat v2.0.9. Bowtie was used for a BWT (Burrows-Wheeler Transformer) algorithm for mapping reads to the genome and Tophat can generate a database of splice junctions based on the gene model annotation file and thus achieve a better mapping result than other non-splice mapping tools. For the quantification of gene expression level, HTSeq V0.6.1 was used to count the read numbers mapped for each gene. The RPKM of each gene was calculated based on the gene read counts mapped to this gene. A differential expression analysis was performed using the DESeq R package (3.18.1). The P values were adjusted using the Benjamini \& Hochberg method. Corrected P-value of 0.05 and absolute foldchange of 2 were set as the threshold for significantly differential expression. Gene Ontology (GO) enrichment analysis of differentially expressed genes, GO enrichment analysis of differentially expressed genes was implemented by the clusterProfiler R package, in which gene length bias was corrected. GO terms with corrected Pvalue less than 0.05 were considered significantly enriched by differential expressed genes.

\subsection{Statistical methods}

Subject-related data were statistically processed by applying SPSS19.0 statistical software. The measurement data were expressed as mean plus or minus standard deviation. And one-way ANOVA with randomized group design was used for comparison between groups, and the LSD method test was used for two-way comparison if the data variance was the same, and the Tamhane's method test was used for two-way comparison if the variance was not the same, and the difference was considered statistically significant at $P<0.05$. The test level was $a=0.05$, and the confidence interval for parameter estimation was $95 \%$.

\section{Results}

\subsection{General information and macro behavioral performance}

Ten ApoE knockout mice and Five C57BL/6J mice of the same strain without deletion were used in the experiment, and 15 mice were entered into the result analysis. Before sampling, the mice in the PDR group showed loss of body hair brightness, lethargy and laziness; the mice in the SKYD group showed arching of the back and curling up, chilling, lethargy and lethargy, lying down and preferring to pile up.

\subsection{Comparison of blood lipid indexes}

By comparing the lipid indexes, compared with the NC group, TG, TC, LDL-C were significantly higher in the PDR group and the SKYD group $(P<0.05)$. Compared with the normal control group, no significant statistical differences were seen in the comparison of HDL-C indexes between the PDR and SKYD groups. No significant statistical differences were seen between the PDR and SKYD groups in the comparison of TG, TC, HDL-C. No statistically significant differences were found in the comparison of TG, TC, HDL-C, LDL-C and LDL-C indexes between the PDR and SKYD groups. See Tables 1 - 4.

\subsection{Comparison of hematoxylin and eosin staining examination}

The aortas in the PDR group and the SKYD group were examined by hematoxylin and eosin staining. And the endothelial surface of the vessels was smooth, and the inner, middle and outer membranes showed clear, and no obvious lipid deposition or lipid streak production was seen. See Figure 2. 


\subsubsection{Analysis of differentially expressed genes in macrophages of mice with different syndromes of dyslipidemia generated by RNA sequencing}

After comparing the DEG expressions in macrophages in dyslipidemia mice with different syndromes, 4142 genes were identified with statistical significance $(P<0.05)$. Among them, 1781 genes were up-regulated and 2361 genes were down-regulated. See Figure 3.

Combined with bioinformatics information, a total of 284 differentially expressed genes were selected in this study for in-depth analysis of content related to vascular endothelial injury. In the comparison of the 74 differentially expressed genes between the PDR group and the SKYD group, the number of reads in the PDR group was statistically higher than that in the SKYD group $(P<0.05)$. See Table 5.

\subsubsection{GO enrichment analysis of differentially expressed genes associated with vascular endothelial injury in macrophages}

On the basis of GO enrichment analysis based on differentially expressed genes, macrophage sequencing data of mice in the PDR group and the SKYD group were analyzed, focusing on the enrichment results of biological processes related to vascular endothelial injury.

The differential pathways that were upregulated in the PDR group compared to the SKYD group mainly included: arachidonic acid metabolic process $\square$ epoxygenase P450 pathway】response to interferon-gamma】cellular response to interferon-beta. See Figure 4.

The differential pathways that were upregulated in the SKYD group compared to the PDR group mainly included: blood vessel morphogenesis $\square$ angiogenesis $\square$ response to growth factor $\rrbracket$ cellular response to growth factor stimulus $\llbracket$ chemotaxis $\square$ taxis. See Figure $\mathbf{5}$.

\section{Discussion}

Dyslipidemia may significantly increase the morbidity and mortality of cardiovascular diseases [[16]]. A study shows that effective control of blood lipid levels may reduce the possibility of relapsed coronary heart disease and its mortality [[17]]. Therefore, proactive diagnosis and treatment for dyslipidemia, the most critical risk factor of atherosclerosis, is essential for decreasing the incidence and mortality of coronary heart disease and cerebral infarction [[18]].

Treatment based on syndrome differentiation is the quintessence of TCM. Syndromes as a distinctive concept in TCM is the core content of the theory and vital evidence for treatment and prescription. TCM treatment exhibits advantages in the management of dyslipidemia [[19]]. The predominant mechanisms encompass inhibiting cholesterol absorption in the intestines and biological synthesis of endogenous lipids, regulating lipoprotein lipase activity and cholesterol transport, promoting the conversion of cholesterol into bile acids and cholesterol emission, and regulatory effects of lipidmetabolism-related transcription factors in TCM drugs [[20]]. Precise discrimination of exact TCM syndromes of dyslipidemia is the prerequisite and basis of TCM treatment. Therefore, TCM syndrome research is a critical link in TCM modernization, wherein biological syndrome research is most significant, providing evidence for the onset and evolution of syndromes and effective mechanisms behind specific interventions for syndromes.

RNA-Seq is a next-generation approach using high throughput sequencing available for low-abundance genomes. It is sensitive for gene structure analysis and gene expression and function assessments to unveil internal molecular mechanisms behind specific biological processes and the pathogenesis of diseases. The features of these genes consist of classic genetic features (nucleotide sequence changes) and epigenetic features (heritable phenotype changes without nucleotide sequence alterations). Our study investigated the syndrome-biological mechanism of dyslipidemia using RNA-Seq.

Our previous serum metabonomics studies of dyslipidemic patients with the PDR syndrome and the SKYD syndrome found that the accumulation of harmful metabolites is the predominant metabolic trait in patients with the PDR syndrome. A lack of protective metabolites is the main metabolic feature in patients with the SKYD syndrome. Further analysis showed that oxidation and inflammatory responses are essential contributors to the different metabolic characteristics between the two syndromes [[21]]. Thus, in-depth study of the SKYD syndrome and the PDR syndrome in dyslipidemia necessitate research on oxidation and inflammatory responses.

Macrophages are pivotal in the formation of atherosclerosis following oxidative stress damage to the vascular endothelium. Endothelial dysfunction and the subsequent oxidative inflammatory reaction is the core pathological mechanism of dyslipidemia. Macrophages are recruited towards endothelial cells, which is an early stimulus of atheromatous plaques formation [[22]] and the important basis of the incidence of atherosclerosis. Based on our previous research, we established the disease-syndrome animal models and explored the characteristics of the aortic endothelial macrophages between the PDR syndrome and the SKYD syndrome of dyslipidemia using RNA-Seq. Our conclusions were as follows.

\section{Quality assessment of the dyslipidemia disease model using serum lipids analysis and HE staining of the aorta, revealing success in disease modeling}

Compared with the NC group, TG, TC, and LDL-C levels significantly increased in mice of the PDR and SKYD groups and exceeded the upper limit of the normal range, in conformity to the diagnostic criterion of dyslipidemia. The HE staining of the aorta revealed a smooth endothelial surface and clear borders between inner, medial, and outer layers in mice of the PDR and SKYD groups, without pronounced lipid accumulation and fatty streaks. These results indicated that there were no pathological manifestations of atherosclerosis in mice of the two groups. The HE staining results implied the satisfactory quality of dyslipidemia modeling. 
However, there were no significant differences in TG, TC, HDL-C, and LDL-C levels between the PDR and SKYD groups. This result suggested that differences in transcriptomic traits and biological results between the two groups using RNA-Seq reflected the characteristics of different syndromes (subtypes).

\section{Quality assessment of the two syndrome models (the PDR models and the SKYD models) through behavioral tests, indicating the feasibility of syndrome modeling}

The characteristics of mice in the PDR group included fat in body shape, reduced brightness of hair, lethargy, slow response, lazy to move, as well as soft, formed, and sticky feces, consistent with clinical manifestations of the PDR syndrome. The behavioral features of mice in the SKYD group incorporated matted hair, slight paw and nail colors, shrinking the body, chilly, fatigue, sleepy, sticking together, low-temperature tail, decrease in food and water intake, loose and watery stools. These were in agreement with clinical manifestations of the SKYD syndrome. These results indicated that the syndrome modeling could simulate typical symptoms of the corresponding syndrome.

3 Transcriptomic data of macrophages showed pro-inflammatory activities in the vascular endothelium in dyslipidemic mice with the PDR syndrome and the SKYD syndrome, consistent with the theory: evil qi leading to the incidence of disease. But this process was achieved via different biological processes in different syndromes, indicating distinct mechanisms of vascular endothelial injury in different syndromes.

\subsection{IFN- $\gamma$ and IFN- $\beta$ expressions were upregulated in macrophages in dyslipidemic mice with the PDR syndrome, promoting endothelial inflammation}

It is known that IFN-y can facilitate the progression of inflammatory diseases, for example, inflammatory bowel disease and atherosclerosis. In-vitro and in-vivo studies have found IFN- $\gamma$ may damage epithelial cells and endothelial barrier integrity [[23]]. IFN- $\gamma$ exerts significant impacts on the biological properties of the vascular endothelial cells. It may initiate vascular remodeling around microvascular endothelial cells [[24]]. Monocyte-derived macrophages are induced by the pro-inflammatory factor IFN- $\gamma$, which are of vital importance during plaque formation. Both IFN- $\gamma$ and macrophages are major players in oxidative stress. Just like other pro-inflammatory factors, IFN- $\gamma$ acts as a significant trigger of the synthesis and release of reactive oxygen species (ROS) [[25]].

IFN- $\beta$ mRNA can effectively induce endothelial chemokine expression [[26]]. It enhances endothelial cell adhesion to eosinophils mainly through upregulating vascular cell adhesion molecule-1 and intercellular adhesion molecule-1 expressions [[27]]. IFN- $\beta$ fuels the formation of macrophage foam cells via SR-A-mediated cholesterol influx and ABCA1-mediated efflux of mechanisms, thus expediting the incidence of atherosclerosis [[28]].

The PDR syndrome is a type of sthenia syndrome. The phlegm evil is both the pathological product and pathogenic factor, impeding the delivery and movement of $q i$, thus resulting in body fluid stagnation and hydrops or damp evil accumulation and phlegm. Finally, the vascular endothelium is impaired.

Our results showed that IFN- $y$ and IFN- $\beta$ expressions in macrophages were upregulated in dyslipidemic mice with the PDR syndrome, significantly higher than the levels in mice with the SKYD syndrome. This finding suggested that vascular endothelial injury induced by IFN- $\gamma$ and IFN- $\beta$ overexpression in vascular endothelial macrophages is a characteristic of the PDR syndrome in dyslipidemia.

\subsection{Macrophage chemotaxis and taxis was enhanced in dyslipidemic mice with the SKYD syndrome, promoting endothelial inflammation}

Macrophages are inflammatory cells, and their accumulation can stimulate cytokine and chemokine release, initiating immune responses and accelerating plaque formation [[29]]. The secreted chemokines infiltrate atherosclerotic plaques at the early stage [[30]]. Despite the role in lipid accumulation, macrophage foam cells also release pro-inflammatory factors and chemokines, further stimulating vascular endothelial cells, fueling vascular endothelial inflammation, and exacerbating the disease [[31]]. A study found that resveratrol could exert a protective effect on the heart via inhibiting endothelial cell migration and monocyte chemotaxis [[32]].

The TCM mechanism of the SKYD syndrome in dyslipidemia refers to spleen-kidney yang deficiency. Furthermore, as the body lacks warmth from yangqi and protection, the vascular endothelium can be easily injured.

Our results showed that macrophage chemotaxis and taxis were significantly enhanced in dyslipidemic mice with the SKYD syndrome, compared with the PDR mice. This finding suggested that vascular endothelial injury induced by enhanced macrophage chemotaxis and taxis were the main characteristic of the SKYD syndrome in dyslipidemia.

The above results showed that different biological processes resulted in vascular endothelial injury in the PDR syndrome and the SKYD syndrome of dyslipidemia, indicating different injury mechanisms of the two syndromes. These results also imply that there exist biological bases behind the pathogenesis of TCM syndromes.

4 Transcriptomic data of macrophages revealed their protection for the vascular endothelium in dyslipidemic mice with the PDR syndrome and the SKYD syndrome. These results coincide with the TCM trait 'the waxing and waning of healthy energy-evil struggles.' But this trait consists of distinct biological processes in different syndromes, showing different vascular protective mechanisms of different syndromes.

4.1 Arachidonic acid metabolic process and epoxygenase P450 pathway levels increased in macrophages in dyslipidemic mice with the PDR syndrome, exerting protection effects on the vascular endothelium 
Growing evidence has shown that AA metabolism is crucial in maintaining vascular homeostasis, closely associated with the occurrence and development of cardiovascular diseases [[33]]. AA is an amphiphilic compound affecting endothelial cell migration without the involvement of receptorspecific signaling, it affects endothelial cell metabolism and membrane viscosity [[34]]. AA metabolic pathways are pivotal in platelet activation and gastric damage [[35]]. Suppressing AA metabolism can further block endothelial cell migration, inducing cell apoptosis [[36]]. A study reported that Panax notoginseng saponins combined with aspirin inhibited platelet activity via enhancing AA metabolism [[37]].

Cytochrome P450 (CYP450) refers to a third pathway for AA metabolism [[38]]. CYP450 metabolites of AA in endothelial cells may impact endothelial function. AA is metabolized by CYP450 and cyclooxygenase (COX) into bioactive eicosanoids, exerting vascular protection effects [[39]]. AA is also metabolized by CYP450 and COX into four regioisomeric epoxyeicosatrienoic acids (EETs) used for bioprotection and cadioprotection [[40]]. EETs have multiple nutritive functions, including the anti-inflammatory effect, in their cadioprotection [[41]]. Decreases in EETs expressions may lead to the onset of cardiovascular diseases and endothelial dysfunction [[42]].

Our analysis showed significant upregulations of arachidonic acid metabolic process and epoxygenase P450 pathway levels in macrophages in dyslipidemic mice with the PDR syndrome versus the SKYD mice. These results indicated that endothelial protection from macrophages via AA and CYP450 overexpressions is another trait of the PDR syndrome in dyslipidemia.

\subsection{Biological process items, including angiogenesis, blood vessel morphogenesis, response to growth factor, and cellular response to growth factor stimulus, whose activities were significantly enhanced for macrophages in dyslipidemic mice with the SKYD syndrome, which facilitated angiogenesis and vascular repair}

Angiogenesis consists of multiple intricate, highly-coordinated processes, wherein endothelial cells with dynamic changes are of great importance [[43]]. Angiogenesis may occur in the pathological environment [[44]] and be initiated by endothelial cell activation. The genetic program of endothelial cells triggers the modulation of angiogenic phenotype. Macrophages are significant regulators for tissue homeostasis, growth, and repair, and morphogenesis. The growing endothelial cells can respond to extracellular signaling molecules, such as extracellular matrix molecules, chemokines, growth factors, and cell adhesion molecules [[45]]. Growth factors and cytokines secreted from macrophages [[46]] may promote the formation of new blood vessels via recruiting new blood vessels and modifying the extracellular matrix [[47]]. Vascular endothelial growth factors (VEGFs) are considered the most robust booster for angiogenesis, increasing the survival of endothelial cells and enhancing mitosis [[48]]. Numerous studies have proven that VEGFs are expressed in macrophages [[49]]. They can stimulate assorted cell functions of endothelial cells via high-affinity binding to two tyrosine kinase receptors, VEGF receptor VEGFR1 and VEGFR2 [[50]].

Our results demonstrated that the top biological process items enriched in macrophages in dyslipidemic mice with the SKYD syndrome were as follows: angiogenesis, blood vessel morphogenesis, response to growth factor, cellular response to growth factor stimulus, whose activities were significantly enhanced versus the the PDR group. This finding suggested that angiogenesis and vascular repair via enhancing macrophage chemotaxis and taxis are another critical feature of the SKYD syndrome in dyslipidemia.

The above results indicated that vascular protection mechanisms are distinct between the PDR syndrome and the SKYD syndrome in dyslipidemia. It further implies different protection mechanisms in the two syndromes. This further proves that there exist biological bases behind the pathogenesis of TCM syndromes.

According to the results in Sections 3 and 4, the vascular endothelial injury was induced by different biological processes in the PDR syndrome and the SKYD syndrome of dyslipidemia. But there also existed angiogenesis and vascular repair in the pathogenetic process. The two opposite processes, injury and repair, are consistent with the TCM rule 'healthy qi and evil qi are struggling throughout the incidence and dynamic development of diseases and syndromes.' As the injury effect outweighs the repairing effect, the state of diseases takes place.

\section{Different transcriptomic characteristics of aortic endothelial macrophages between dyslipidemic mice with the PDR syndrome and the SKYD syndrome, which are manifested by distinct biological control processes during both harmful and protective biological processes, indicating different biological bases behind different syndromes of the same disease and providing biological evidence for the TCM theory 'treating the same disease with different treatments' (Figure 6)}

In the TCM clinic, western medicine diagnosis is often combined with TCM syndrome diagnosis for the management of a disease. Research about the association between western diseases and TCM syndromes is believed to be one of the most important steps for modern TCM diagnostics studies. Based on the confirmation of a western disease, disease-syndrome research can not only elucidate the biological bases for TCM differentiation but also help push innovative research on 'disease-syndrome-therapy-formula' and thereby provide precise and rational treatment.

Previous TCM syndrome research emphasizes harmful factors in a syndrome scenario, without much attention to the body's self-protection in this process. Our study did both, focusing on the analysis of harmful factors and protective factors, which is an innovative dimension. The unity of opposites, protective and harmful effects, is achieved by dynamic balancing between healthy qi and evil qi through mutual conflicts, mutual restriction, and mutual repulsion. Diseases can occur when evil qi outstrips healthy $q i$, just as a TCM rule saying 'when there is sufficient healthy qi inside, pathogenic factors have no way to invade the body; where pathogenic factors accumulate, the parts of the body must be deficient in the healthy qi'. TCM treatment should be implemented based on accurate discrimination of diseases and syndromes and hit the mark by correcting the imbalance between healthy qi and evil qi through prescriptions and formulas. 


\section{Conclusions}

Our transcriptomic analysis of aortic endothelial macrophages in dyslipidemic mice with the PDR syndrome and the SKYD syndrome showed the results as follows. First, differentially expressed genes were identified between dyslipidemic mice with the PDR syndrome and the SKYD syndrome, proving different biological mechanisms during the pathogenesis of different syndromes, from the perspective of syndrome research. Second, there existed different biological processes between the PDR syndrome and the SKYD syndrome of dyslipidemia, including harmful and protective biological processes. When evil qi invades the body to produce harmful effects, healthy qialso responds to it and thereby generates protective responses in a syndrome scenario, which agrees with the TCM rule 'healthy qi and evil qi are struggling throughout the incidence and dynamic development of diseases and syndromes.' Therefore, the occurrence of syndromes is a result of healthy energy-evil struggles. That is why TCM treatment for one disease with various therapies and formulas can achieve satisfactory efficacy, which may be attributed to different drugs targeting different biological processes. Our work offers biological mechanisms for the TCM theories 'treating different syndromes with different treatments' and 'formula corresponding to the syndrome.' Third, though patients may be diagnosed with the same disease dyslipidemia, different formulas should be selected according to their syndromes, considering distinct biological processes during the PDR syndrome and the SKYD syndrome. Our study has demonstrated the biological evidence behind 'treating the same disease with different treatments' in TCM, embodying the scientificity of 'treatment based on syndrome differentiation.'

\section{Abbreviations}

TCM Traditional Chinese Medicine

PDR syndrome Phlegm-Dampness Retention syndrome

SKYD syndrome Spleen and Kidney Yang Deficiency syndrome

DEGs DEG analysis

\section{Declarations}

\section{Ethics approval and consent to participate}

The ethics of this study was approved by the animal ethics review committee of the Institute of Basic Theories of Chinese medicine, Chinese Academy of Chinese Medical Sciences, approval no. 201908006 (Beijing, China). All the. methods were performed in accordance with the relevant guidelines and regulations.

\section{Consent for publication}

All authors read and approved the final manuscript.

\section{Availability of data and materials}

Please contact author for data requests.

\section{Competing interests}

The authors declare that the research was conducted in the absence of any commercial or financial relationships that could be construed as a potential conflict of interest.This manuscript/data, or parts thereof, has not been submitted for possible publication to another journal or that the work has previously been published elsewhere.

\section{Author Contributions}

$\mathrm{XX}$ and JC conceived and designed the study. JC, CY, SZ and PL performed the modeling and evaluation of animal models of diseases and syndromes. JC, TW, ZY and BX performed the sampling and macrophage screening.JC, CY, ZY, BX, LP and XX acquired the data. JC, CY, ZY and BX analyzed and interpreted data. JC and CY drafted the manuscript. PL and ZY critically revised the manuscript for important intellectual content. All authors read and approved the final manuscript. 
Funding

This study was supported by the National Natural Science Foundation of China (grant number 82004237), Fundamental Research Funds for the Central Universities (grant number 2018-JYBZZ-JS182) , Beijing University of Chinese Medicine Xin-ao Award Fund (grant number 2018-XAJLJJ-026) and National Natural Science Foundation of China (grant number 81704133).

\section{Acknowledgments}

The authors would like to thank Prof. Tongjin Zhao (Institute of Metabolism \& Integrative Biology (IMIB), Fudan University, Shanghai, China) for technical assistance with this study.

\section{References}

[1]. Li Z, Zhao T, Tan X, Lei S, Huang L, Yang L: Polymorphisms in PCSK9, LDLR, BCM01, SLC12A3, and KCNJ1 are Associated with Serum Lipid Profile in Chinese Han Population. Int J Environ Res Public Health 2019, 16(17): 3207.

[2]. Chu J GR, Zhao S, et al. : Guidelines for the prevention and treatment of dyslipidemia in Chinese adults (revised edition in 2016). Chinese Circulation Journal 2016, 31(10):937-953.

[3]. Sarzynski MA, Schuna JM, Jr., Carnethon MR, Jacobs DR, Jr., Lewis CE, Quesenberry CP, Jr., Sidney S, Schreiner PJ, Sternfeld B: Association of Fitness With Incident Dyslipidemias Over 25 Years in the Coronary Artery Risk Development in Young Adults Study. Am J Prev Med 2015, 49(5):745-752.

[4]. Pantos J, Efstathopoulos E, Katritsis DG: Vascular wall shear stress in clinical practice. Curr Vasc Pharmaco/ 2007, 5(2):113-119.

[5]. Ciccone MM, Miniello V, Marchioli R, Scicchitano P, Cortese F, Palumbo V, Primitivo SG, Sassara M, Ricci G, Carbonara S et al: Morphological and functional vascular changes induced by childhood obesity. Eur J Cardiovasc Prev Rehabil 2011, 18(6):831-835.

[6]. Stone NJ, Robinson JG, Lichtenstein AH, Bairey Merz CN, Blum CB, Eckel RH, Goldberg AC, Gordon D, Levy D, Lloyd-Jones DM et al: 2013 ACC/AHA guideline on the treatment of blood cholesterol to reduce atherosclerotic cardiovascular risk in adults: a report of the American College of Cardiology/American Heart Association Task Force on Practice Guidelines. Circulation 2014, 129(25 Suppl 2):S1-45.

[7]. He C, Fu P, Zhang K, Xia Q, Yang Y, Xie L: Chinese herbal medicine for dyslipidemia: protocol for a systematic review and meta-analysis. Medicine (Baltimore) 2018, 97(44):e13048.

[8]. Eelen G, Treps L, Li X, Carmeliet P: Basic and Therapeutic Aspects of Angiogenesis Updated. Circ Res 2020, 127(2):310-329.

[9]. Scicchitano P, Cortese F, Gesualdo M, De Palo M, Massari F, Giordano P, Ciccone MM: The role of endothelial dysfunction and oxidative stress in cerebrovascular diseases. Free Radic Res 2019, 53(6):579-595.

[10]. Chen J YC, Yang Z, et al: The correlation between the traditional Chinese medicine (TCM) syndrome and the concentration of adiponectin and peroxynitrite in dyslipidemia patients. European Journal of Integrative Medicine 2016, 8(6):973-979.

[11]. Davies LC, Taylor PR: Tissue-resident macrophages: then and now. Immunology 2015, 144(4):541-548.

[12]. Pesce JT, Ramalingam TR, Mentink-Kane MM, Wilson MS, El Kasmi KC, Smith AM, Thompson RW, Cheever AW, Murray PJ, Wynn TA: Arginase-1expressing macrophages suppress Th2 cytokinedriven inflammation and fibrosis. PLoS Pathog 2009, 5(4):e1000371.

[13]. Moore EM, West JL: Harnessing Macrophages for Vascularization in Tissue Engineering. Ann Biomed Eng 2019, 47(2):354-365.

[14]. Yamada Y, Doi T, Hamakubo T, Kodama T: Scavenger receptor family proteins: roles for atherosclerosis, host defence and disorders of the central nervous system. Cell Mol Life Sci 1998, 54(7):628-640.

[15]. Jiang TT, Li JC: Review on the systems biology research of Yin-deficiency-heat syndrome in traditional Chinese medicine. Anat Rec (Hoboken) 2020. doi: 10.1002/ar.24354.

[16]. Lee JS, Chang PY, Zhang Y, Kizer JR, Best LG, Howard BV: Triglyceride and HDL-C Dyslipidemia and Risks of Coronary Heart Disease and Ischemic Stroke by Glycemic Dysregulation Status: The Strong Heart Study. Diabetes Care 2017, 40(4):529-537.

[17]. Mozaffarian D, Benjamin EJ, Go AS, Arnett DK, Blaha MJ, Cushman M, de Ferranti S, Despres JP, Fullerton HJ, Howard VJ et al: Heart disease and stroke statistics-2015 update: a report from the American Heart Association. Circulation 2015, 131(4):e29-322.

[18]. Rhee EJ, Kim HC, Kim JH, Lee EY, Kim BJ, Kim EM, Song Y, Lim JH, Kim HJ, Choi S et al: 2018 Guidelines for the management of dyslipidemia in Korea. Korean J Intern Med 2019, 34(5):1171. 
[19]. Sham TT, Chan CO, Wang YH, Yang JM, Mok DK, Chan SW: A review on the traditional Chinese medicinal herbs and formulae with hypolipidemic effect. Biomed Res Int 2014, 2014:925302.

[20]. Bei WJ, Guo J, Wu HY, Cao Y: Lipid-regulating effect of traditional chinese medicine: mechanisms of actions. Evid Based Complement Alternat Med 2012, 2012:970635.

[21]. Chen J, Ye C, Hu X, Huang C, Yang Z, Li P, Wu A, Xue X, Lin D, Yang H: Serum metabolomics model and its metabolic characteristics in patients with different syndromes of dyslipidemia based on nuclear magnetic resonance. J Pharm Biomed Anal 2019, 167:100-113.

[22]. Tiwari RL, Singh V, Barthwal MK: Macrophages: an elusive yet emerging therapeutic target of atherosclerosis. Med Res Rev 2008, 28(4):483-544.

[23]. Ng CT, Fong LY, Sulaiman MR, Moklas MA, Yong YK, Hakim MN, Ahmad Z: Interferon-Gamma Increases Endothelial Permeability by Causing Activation of p38 MAP Kinase and Actin Cytoskeleton Alteration. J Interferon Cytokine Res 2015, 35(7):513-522.

[24]. Chrobak I, Lenna S, Stawski L, Trojanowska M: Interferon-gamma promotes vascular remodeling in human microvascular endothelial cells by upregulating endothelin (ET)-1 and transforming growth factor (TGF) beta2. J Cell Physiol 2013, 228(8):1774-1783.

[25]. Schroecksnadel K, Frick B, Winkler C, Fuchs D: Crucial role of interferon-gamma and stimulated macrophages in cardiovascular disease. Curr VasC Pharmacol 2006, 4(3):205-213.

[26]. Buttmann M, Berberich-Siebelt F, Serfling E, Rieckmann P: Interferon-beta is a potent inducer of interferon regulatory factor-1/2-dependent IP10/CXCL10 expression in primary human endothelial cells. J Vasc Res 2007, 44(1):51-60.

[27]. Kobayashi T, Takaku Y, Yokote A, Miyazawa H, Soma T, Hagiwara K, Kanazawa M, Nagata M: Interferon-beta augments eosinophil adhesioninducing activity of endothelial cells. Eur Respir J 2008, 32(6):1540-1547.

[28]. Boshuizen MC, Hoeksema MA, Neele AE, van der Velden S, Hamers AA, Van den Bossche J, Lutgens E, de Winther MP: Interferon-beta promotes macrophage foam cell formation by altering both cholesterol influx and efflux mechanisms. Cytokine 2016, 77:220-226.

[29]. Liehn EA, Zernecke A, Postea O, Weber C: Chemokines: inflammatory mediators of atherosclerosis. Arch Physiol Biochem 2006, 112(4-5):229-238.

[30]. Weber C, Zernecke A, Libby P: The multifaceted contributions of leukocyte subsets to atherosclerosis: lessons from mouse models. Nat Rev Immunol 2008, 8(10):802-815.

[31]. Kleemann R, Zadelaar S, Kooistra T: Cytokines and atherosclerosis: a comprehensive review of studies in mice. Cardiovasc Res 2008, 79(3):360376.

[32]. Cicha I, Regler M, Urschel K, Goppelt-Struebe M, Daniel WG, Garlichs CD: Resveratrol inhibits monocytic cell chemotaxis to MCP-1 and prevents spontaneous endothelial cell migration through Rho kinase-dependent mechanism. J Atheroscler Thromb 2011, 18(12):1031-1042.

[33]. Xue SS, He JL, Zhang X, Liu YJ, Xue FX, Wang CJ, Ai D, Zhu Y: Metabolomic analysis revealed the role of DNA methylation in the balance of arachidonic acid metabolism and endothelial activation. Biochim Biophys Acta 2015, 1851(10):1317-1326.

[34]. Jensen LD, Hansen AJ, Lundbaek JA: Regulation of endothelial cell migration by amphiphiles - are changes in cell membrane physical properties involved? Angiogenesis 2007, 10(1):13-22.

[35]. Rukoyatkina N, Shpakova V, Panteleev M, Kharazova A, Gambaryan S, Geiger J: Multifaceted effects of arachidonic acid and interaction with cyclic nucleotides in human platelets. Thromb Res 2018, 171:22-30.

[36]. Jantke J, Ladehoff M, Kurzel F, Zapf S, Kim E, Giese A: Inhibition of the arachidonic acid metabolism blocks endothelial cell migration and induces apoptosis. Acta Neurochir (Wien) 2004, 146(5):483-494.

[37]. Wang W, Yang L, Song L, Guo M, Li C, Yang B, Wang M, Kou N, Gao J, Qu H et al: Combination of Panax notoginseng saponins and aspirin potentiates platelet inhibition with alleviated gastric injury via modulating arachidonic acid metabolism. Biomed Pharmacother 2021, $134: 111165$.

[38]. Roman RJ: P-450 metabolites of arachidonic acid in the control of cardiovascular function. Physiol Rev 2002, 82(1):131-185.

[39]. Spiecker M, Liao JK: Vascular protective effects of cytochrome p450 epoxygenase-derived eicosanoids. Arch Biochem Biophys 2005, 433(2):413420.

[40]. Xu X, Zhang XA, Wang DW: The roles of CYP450 epoxygenases and metabolites, epoxyeicosatrienoic acids, in cardiovascular and malignant diseases. Adv Drug Deliv Rev 2011, 63(8):597-609.

[41]. Node K, Huo Y, Ruan X, Yang B, Spiecker M, Ley K, Zeldin DC, Liao JK: Anti-inflammatory properties of cytochrome P450 epoxygenase-derived eicosanoids. Science 1999, 285(5431):1276-1279. 
[42]. Bellien J, lacob M, Remy-Jouet I, Lucas D, Monteil C, Gutierrez L, Vendeville C, Dreano Y, Mercier A, Thuillez C et al: Epoxyeicosatrienoic acids contribute with altered nitric oxide and endothelin-1 pathways to conduit artery endothelial dysfunction in essential hypertension. Circulation 2012 , 125(10):1266-1275.

[43]. Potente M, Gerhardt H, Carmeliet P: Basic and therapeutic aspects of angiogenesis. Cell 2011, 146(6):873-887.

[44]. Alvarez Arroyo MV, Caramelo C, Angeles Castilla M, Gonzalez Pacheco FR, Martin O, Arias J: Role of vascular endothelial growth factor in the response to vessel injury. Kidney Int Supp/ 1998, 68:S7-9.

[45]. Jeong HW, Hernandez-Rodriguez B, Kim J, Kim KP, Enriquez-Gasca R, Yoon J, Adams S, Scholer HR, Vaquerizas JM, Adams RH: Transcriptional regulation of endothelial cell behavior during sprouting angiogenesis. Nat Commun 2017, 8(1):726.

[46]. Lucas T, Waisman A, Ranjan R, Roes J, Krieg T, Muller W, Roers A, Eming SA: Differential roles of macrophages in diverse phases of skin repair. J Immunol 2010, 184(7):3964-3977.

[47]. Corliss BA AM, Munson JM,et al: Macrophages: An Inflammatory Link Between Angiogenesis and Lymphangiogenesis. Microcirculation 2016, 23(2):95-121.

[48]. Dvorak HF: Angiogenesis: update 2005. J Thromb Haemost 2005, 3(8):1835-1842.

[49]. Guo L, Akahori H, Harari E, Smith SL, Polavarapu R, Karmali V, Otsuka F, Gannon RL, Braumann RE, Dickinson MH et al: CD163+ macrophages promote angiogenesis and vascular permeability accompanied by inflammation in atherosclerosis. J Clin Invest 2018, 128(3):1106-1124.

[50]. Zachary I: VEGF signalling: integration and multi-tasking in endothelial cell biology. Biochem Soc Trans 2003, 31(Pt 6):1171-1177.

\section{Tables}

\begin{tabular}{|lc|}
\hline Table 1 Total cholesterol index in 3 groups of mice (mean \pm standard deviation) \\
\hline Group & TG index \\
\hline PDR group & $23.32 \pm 2.33^{\mathrm{a}}$ \\
\hline SKYD group & $29.32 \pm 6.32^{\mathrm{bc}}$ \\
\hline NC group & $2.38 \pm 0.38$ \\
\hline F & 65.82 \\
\hline$P$ & 0.000 \\
\hline
\end{tabular}

Note: a was compared with NC group, $P<0.05$; b was compared with NC group, $P<0.05$; c was compared with PDR group, $P>0.05$;

\begin{tabular}{|lc|}
\hline \multicolumn{2}{|l|}{ Table 2 Triglycerides index in 3 groups of mice (mean \pm standard deviation) } \\
\hline Group & TC index \\
\hline PDR group & $2.96 \pm 0.11^{\mathrm{a}}$ \\
\hline SKYD group & $4.71 \pm 1.15^{\mathrm{bc}}$ \\
\hline NC group & $0.80 \pm 0.79$ \\
\hline F & 43.07 \\
\hline$P$ & 0.000 \\
\hline
\end{tabular}

Note: a was compared with NC group, $P<0.05$; b was compared with NC group, $P<0.05$; c was compared with PDR group, $P>0.05$;

Table 3 HDL-C index in 3 groups of mice (mean \pm standard deviation) 


\begin{tabular}{|lc|}
\hline Group & HDL-C index \\
PDR group & $5.17 \pm 2.15$ \\
\hline SKYD group & $3.03 \pm 1.92^{\mathrm{C}}$ \\
NC group & $3.55 \pm 0.68$ \\
\hline F & 2.13 \\
\hline$P$ & 0.162 \\
\hline
\end{tabular}

Note: c was compared with PDR group, $P>0.05$

\begin{tabular}{|lc|}
\hline \multicolumn{2}{|c|}{ Table 4 LDL-C index in 3 groups of mice (mean \pm standard deviation) } \\
\hline Group & LDL-C index \\
\hline PDR group & $2.54 \pm 0.22^{a}$ \\
\hline SKYD group & $3.60 \pm 1.39$ bc \\
\hline NC group & $0.89 \pm 0.12$ \\
\hline F & 13.88 \\
\hline P & 0.001 \\
\hline
\end{tabular}

Note: a was compared with NC group, $P<0.05$; b was compared with NC group, $P<0.05$; c was compared with PDR group, $P>0.05$;

Table 5 Injury or protection of macrophages and vascular endothelium in mice with different syndromes of dyslipidemia related differentially expressed genes 


\begin{tabular}{|c|c|c|c|c|c|c|c|}
\hline Gene ID & $\begin{array}{l}\text { PDR } \\
\text { count }\end{array}$ & $\begin{array}{l}\text { SKYD_ } \\
\text { count }\end{array}$ & $\begin{array}{l}p \\
\text { value }\end{array}$ & Gene ID & $\begin{array}{l}\text { PDR } \\
\text { count }\end{array}$ & $\begin{array}{l}\text { SKYD_ } \\
\text { count }\end{array}$ & $\begin{array}{l}p \\
\text { value }\end{array}$ \\
\hline ENSMUSG00000022445 & 323 & 47 & 0.00 & ENSMUSG00000026104 & 1169 & 355 & 0.00 \\
\hline ENSMUSG00000032487 & 57 & 2 & 0.00 & ENSMUSG00000035042 & 2000 & 645 & 0.00 \\
\hline ENSMUSG00000025002 & 73 & 8 & 0.00 & ENSMUSG00000018930 & 875 & 280 & 0.00 \\
\hline ENSMUSG00000060407 & 456 & 100 & 0.00 & ENSMUSG00000035373 & 2723 & 943 & 0.00 \\
\hline ENSMUSG00000068086 & 866 & 203 & 0.00 & ENSMUSG00000055170 & 39 & 6 & 0.00 \\
\hline ENSMUSG00000025479 & 3178 & 776 & 0.00 & ENSMUSG00000022504 & 1244 & 432 & 0.00 \\
\hline ENSMUSG00000060613 & 1120 & 279 & 0.00 & ENSMUSG00000024411 & 16 & 0 & 0.00 \\
\hline ENSMUSG00000052974 & 706 & 175 & 0.00 & ENSMUSG00000029298 & 686 & 256 & 0.00 \\
\hline ENSMUSG00000094806 & 514 & 128 & 0.00 & ENSMUSG00000041515 & 5566 & 2360 & 0.00 \\
\hline ENSMUSG00000003053 & 3283 & 926 & 0.00 & ENSMUSG00000074151 & 241 & 96 & 0.00 \\
\hline ENSMUSG00000025197 & 100 & 20 & 0.00 & ENSMUSG00000000791 & 204 & 83 & 0.00 \\
\hline ENSMUSG00000054827 & 1406 & 415 & 0.00 & ENSMUSG00000049093 & 19 & 3 & 0.00 \\
\hline ENSMUSG00000092008 & 30 & 2 & 0.00 & ENSMUSG00000030966 & 800 & 382 & 0.01 \\
\hline ENSMUSG00000030483 & 61 & 13 & 0.00 & ENSMUSG00000026866 & 485 & 240 & 0.01 \\
\hline ENSMUSG00000005547 & 252 & 82 & 0.00 & ENSMUSG00000054072 & 5433 & 269 & 0.00 \\
\hline ENSMUSG00000033174 & 211 & 67 & 0.00 & ENSMUSG00000078921 & 5214 & 719 & 0.00 \\
\hline ENSMUSG00000042248 & 216 & 71 & 0.00 & ENSMUSG00000068606 & 130 & 9 & 0.00 \\
\hline ENSMUSG00000068083 & 54 & 13 & 0.00 & ENSMUSG00000073555 & 1016 & 177 & 0.00 \\
\hline ENSMUSG00000025004 & 57 & 14 & 0.00 & ENSMUSG00000090942 & 146 & 20 & 0.00 \\
\hline ENSMUSG00000074882 & 299 & 123 & 0.00 & ENSMUSG00000048852 & 104 & 15 & 0.00 \\
\hline ENSMUSG00000062624 & 200 & 82 & 0.00 & ENSMUSG00000046879 & 10788 & 2545 & 0.00 \\
\hline ENSMUSG00000067225 & 324 & 140 & 0.00 & ENSMUSG00000074896 & 2248 & 623 & 0.00 \\
\hline ENSMUSG00000024292 & 105 & 40 & 0.00 & ENSMUSG00000078920 & 14916 & 4448 & 0.00 \\
\hline ENSMUSG00000061740 & 96 & 40 & 0.01 & ENSMUSG00000078853 & 6044 & 1992 & 0.00 \\
\hline ENSMUSG00000057666 & 4375 & 77 & 0.00 & ENSMUSG00000058163 & 432 & 208 & 0.01 \\
\hline ENSMUSG00000040264 & 4138 & 114 & 0.00 & ENSMUSG00000004814 & 326 & 5501 & 0.00 \\
\hline ENSMUSG00000022126 & 1783 & 66 & 0.00 & ENSMUSG00000076431 & 19 & 455 & 0.00 \\
\hline ENSMUSG00000078922 & 3013 & 362 & 0.00 & ENSMUSG00000040152 & 392 & 5031 & 0.00 \\
\hline ENSMUSG00000028270 & 7705 & 1058 & 0.00 & ENSMUSG00000022309 & 0 & 90 & 0.00 \\
\hline ENSMUSG00000004296 & 121 & 13 & 0.00 & ENSMUSG00000031785 & 0 & 88 & 0.00 \\
\hline ENSMUSG00000028268 & 6736 & 1336 & 0.00 & ENSMUSG00000036856 & 0 & 77 & 0.00 \\
\hline ENSMUSG00000069874 & 1240 & 268 & 0.00 & ENSMUSG00000019997 & 1 & 89 & 0.00 \\
\hline ENSMUSG00000105096 & 93 & 13 & 0.00 & ENSMUSG00000018593 & 187 & 1817 & 0.00 \\
\hline ENSMUSG00000104713 & 389 & 83 & 0.00 & ENSMUSG00000022018 & 848 & 7779 & 0.00 \\
\hline ENSMUSG00000035385 & 5307 & 1309 & 0.00 & ENSMUSG00000022469 & 66 & 674 & 0.00 \\
\hline ENSMUSG00000030895 & 4179 & 1033 & 0.00 & ENSMUSG00000061878 & 15 & 217 & 0.00 \\
\hline ENSMUSG00000060550 & 12164 & 3456 & 0.00 & ENSMUSG00000031740 & 4 & 98 & 0.00 \\
\hline ENSMUSG00000079363 & 1587 & 447 & 0.00 & ENSMUSG00000010660 & 6 & 114 & 0.00 \\
\hline ENSMUSG00000018899 & 5290 & 1555 & 0.00 & ENSMUSG00000038545 & 1 & 69 & 0.00 \\
\hline ENSMUSG00000105504 & 237 & 63 & 0.00 & ENSMUSG00000006445 & 37 & 359 & 0.00 \\
\hline
\end{tabular}


Page 14/24 


\begin{tabular}{|c|c|c|c|c|c|c|c|}
\hline Gene ID & $\begin{array}{l}\text { PDR } \\
\text { count }\end{array}$ & $\begin{array}{l}\text { SKYD_ } \\
\text { count }\end{array}$ & $\begin{array}{l}p \\
\text { value }\end{array}$ & Gene ID & $\begin{array}{l}\text { PDR } \\
\text { count }\end{array}$ & $\begin{array}{l}\text { SKYD_ } \\
\text { count }\end{array}$ & $\begin{array}{l}p \\
\text { value }\end{array}$ \\
\hline ENSMUSG00000032011 & 149 & 1190 & 0.00 & ENSMUSG00000044317 & 0 & 14 & 0.00 \\
\hline ENSMUSG00000004951 & 310 & 2300 & 0.00 & ENSMUSG00000003032 & 70 & 229 & 0.00 \\
\hline ENSMUSG00000029373 & 3690 & 26049 & 0.00 & ENSMUSG00000018500 & 418 & 1239 & 0.00 \\
\hline ENSMUSG00000024486 & 0 & 53 & 0.00 & ENSMUSG00000023031 & 71 & 227 & 0.00 \\
\hline ENSMUSG00000050711 & 0 & 49 & 0.00 & ENSMUSG00000019256 & 52 & 170 & 0.00 \\
\hline ENSMUSG00000025355 & 290 & 1978 & 0.00 & ENSMUSG00000020063 & 46 & 153 & 0.00 \\
\hline ENSMUSG00000037095 & 2041 & 13391 & 0.00 & ENSMUSG00000049130 & 1852 & 5261 & 0.00 \\
\hline ENSMUSG00000037362 & 0 & 44 & 0.00 & ENSMUSG00000022836 & 0 & 13 & 0.00 \\
\hline ENSMUSG00000049791 & 24 & 201 & 0.00 & ENSMUSG00000010175 & 0 & 13 & 0.00 \\
\hline ENSMUSG00000034353 & 1199 & 6646 & 0.00 & ENSMUSG00000045930 & 0 & 13 & 0.00 \\
\hline ENSMUSG00000067336 & 1 & 44 & 0.00 & ENSMUSG00000032494 & 0 & 13 & 0.00 \\
\hline ENSMUSG00000037411 & 0 & 33 & 0.00 & ENSMUSG00000028195 & 12 & 54 & 0.00 \\
\hline ENSMUSG00000050953 & 0 & 33 & 0.00 & ENSMUSG00000005413 & 1251 & 3480 & 0.00 \\
\hline ENSMUSG00000002603 & 72 & 381 & 0.00 & ENSMUSG00000054364 & 185 & 535 & 0.00 \\
\hline ENSMUSG00000016494 & 58 & 313 & 0.00 & ENSMUSG00000022475 & 96 & 284 & 0.00 \\
\hline ENSMUSG00000044337 & 304 & 1399 & 0.00 & ENSMUSG00000026836 & 0 & 12 & 0.00 \\
\hline ENSMUSG00000001300 & 35 & 189 & 0.00 & ENSMUSG00000073599 & 10 & 43 & 0.00 \\
\hline ENSMUSG00000019929 & 1375 & 5747 & 0.00 & ENSMUSG00000030069 & 4 & 26 & 0.00 \\
\hline ENSMUSG00000004791 & 0 & 24 & 0.00 & ENSMUSG00000031565 & 198 & 544 & 0.00 \\
\hline ENSMUSG00000021611 & 0 & 24 & 0.00 & ENSMUSG00000017417 & 75 & 214 & 0.00 \\
\hline ENSMUSG00000034881 & 247 & 976 & 0.00 & ENSMUSG00000031503 & 2 & 19 & 0.00 \\
\hline ENSMUSG00000028108 & 7728 & 28858 & 0.00 & ENSMUSG00000022505 & 2 & 19 & 0.00 \\
\hline ENSMUSG00000038742 & 16 & 89 & 0.00 & ENSMUSG00000042745 & 416 & 1089 & 0.00 \\
\hline ENSMUSG00000040289 & 0 & 20 & 0.00 & ENSMUSG00000068196 & 0 & 11 & 0.00 \\
\hline ENSMUSG00000025473 & 497 & 1724 & 0.00 & ENSMUSG00000042258 & 0 & 11 & 0.00 \\
\hline ENSMUSG00000001827 & 0 & 19 & 0.00 & ENSMUSG00000031465 & 0 & 11 & 0.00 \\
\hline ENSMUSG00000031250 & 0 & 18 & 0.00 & ENSMUSG00000026883 & 14 & 54 & 0.00 \\
\hline ENSMUSG00000045005 & 0 & 17 & 0.00 & ENSMUSG00000033191 & 1 & 14 & 0.00 \\
\hline ENSMUSG00000044562 & 0 & 17 & 0.00 & ENSMUSG00000031613 & 877 & 2203 & 0.00 \\
\hline ENSMUSG00000006235 & 0 & 17 & 0.00 & ENSMUSG00000024241 & 15 & 55 & 0.00 \\
\hline ENSMUSG00000000392 & 0 & 17 & 0.00 & ENSMUSG00000015468 & 11 & 41 & 0.00 \\
\hline ENSMUSG00000020676 & 18 & 84 & 0.00 & ENSMUSG00000024087 & 0 & 10 & 0.00 \\
\hline ENSMUSG00000022893 & 0 & 16 & 0.00 & ENSMUSG00000055254 & 0 & 10 & 0.00 \\
\hline ENSMUSG00000027996 & 0 & 16 & 0.00 & ENSMUSG00000023885 & 0 & 10 & 0.00 \\
\hline ENSMUSG00000015957 & 80 & 277 & 0.00 & ENSMUSG00000049001 & 0 & 10 & 0.00 \\
\hline ENSMUSG00000027276 & 4 & 32 & 0.00 & ENSMUSG00000028868 & 338 & 835 & 0.00 \\
\hline ENSMUSG00000069763 & 16 & 73 & 0.00 & ENSMUSG00000030123 & 43 & 121 & 0.01 \\
\hline ENSMUSG00000001761 & 26 & 101 & 0.00 & ENSMUSG00000016933 & 51 & 140 & 0.01 \\
\hline ENSMUSG00000057789 & 9 & 46 & 0.00 & ENSMUSG00000022528 & 153 & 382 & 0.01 \\
\hline ENSMUSG00000030605 & 213 & 650 & 0.00 & ENSMUSG00000031902 & 227 & 558 & 0.01 \\
\hline
\end{tabular}


Page 16/24 


\begin{tabular}{|c|c|c|c|c|c|c|c|}
\hline Gene ID & $\begin{array}{l}\text { PDR } \\
\text { count }\end{array}$ & $\begin{array}{l}\text { SKYD_ } \\
\text { count }\end{array}$ & $\begin{array}{l}p \\
\text { value }\end{array}$ & Gene ID & $\begin{array}{l}\text { PDR } \\
\text { count }\end{array}$ & $\begin{array}{l}\text { SKYD_ } \\
\text { count }\end{array}$ & $\begin{array}{l}p \\
\text { value }\end{array}$ \\
\hline ENSMUSG00000022425 & 47 & 129 & 0.01 & ENSMUSG00000014932 & 0 & 18 & 0.00 \\
\hline ENSMUSG00000024290 & 275 & 672 & 0.01 & ENSMUSG00000031451 & 85 & 308 & 0.00 \\
\hline ENSMUSG00000027665 & 76 & 194 & 0.01 & ENSMUSG00000024526 & 7 & 44 & 0.00 \\
\hline ENSMUSG00000006311 & 0 & 9 & 0.01 & ENSMUSG00000041120 & 175 & 585 & 0.00 \\
\hline ENSMUSG00000016458 & 0 & 9 & 0.01 & ENSMUSG00000027500 & 0 & 16 & 0.00 \\
\hline ENSMUSG00000026586 & 0 & 9 & 0.01 & ENSMUSG00000029096 & 56 & 200 & 0.00 \\
\hline ENSMUSG00000035458 & 0 & 9 & 0.01 & ENSMUSG00000015619 & 42 & 156 & 0.00 \\
\hline ENSMUSG00000031963 & 0 & 9 & 0.01 & ENSMUSG00000025351 & 1157 & 3589 & 0.00 \\
\hline ENSMUSG00000057722 & 0 & 9 & 0.01 & ENSMUSG00000009406 & 35 & 131 & 0.00 \\
\hline ENSMUSG00000027985 & 279 & 656 & 0.01 & ENSMUSG00000045817 & 241 & 759 & 0.00 \\
\hline ENSMUSG00000004328 & 4 & 21 & 0.01 & ENSMUSG00000022817 & 296 & 921 & 0.00 \\
\hline ENSMUSG00000028763 & 4 & 21 & 0.01 & ENSMUSG00000021253 & 5 & 34 & 0.00 \\
\hline ENSMUSG00000020458 & 156 & 372 & 0.01 & ENSMUSG00000021943 & 0 & 14 & 0.00 \\
\hline ENSMUSG00000026043 & 122 & 291 & 0.01 & ENSMUSG00000026842 & 27 & 101 & 0.00 \\
\hline ENSMUSG00000026193 & 2529 & 9983 & 0.00 & ENSMUSG00000022521 & 80 & 252 & 0.00 \\
\hline ENSMUSG00000000094 & 0 & 9 & 0.01 & ENSMUSG00000029050 & 46 & 153 & 0.00 \\
\hline ENSMUSG00000023078 & 153 & 2061 & 0.00 & ENSMUSG00000020919 & 495 & 1411 & 0.00 \\
\hline ENSMUSG00000049907 & 0 & 103 & 0.00 & ENSMUSG00000038400 & 78 & 240 & 0.00 \\
\hline ENSMUSG00000021319 & 0 & 71 & 0.00 & ENSMUSG00000038872 & 7 & 36 & 0.00 \\
\hline ENSMUSG00000003665 & 0 & 68 & 0.00 & ENSMUSG00000055653 & 39 & 125 & 0.00 \\
\hline ENSMUSG00000036699 & 0 & 62 & 0.00 & ENSMUSG00000044674 & 16 & 63 & 0.00 \\
\hline ENSMUSG00000033295 & 12 & 168 & 0.00 & ENSMUSG00000006958 & 0 & 12 & 0.00 \\
\hline ENSMUSG00000024544 & 73 & 516 & 0.00 & ENSMUSG00000020176 & 0 & 12 & 0.00 \\
\hline ENSMUSG00000029661 & 21 & 187 & 0.00 & ENSMUSG00000011096 & 189 & 520 & 0.00 \\
\hline ENSMUSG00000025854 & 317 & 1960 & 0.00 & ENSMUSG00000024940 & 16 & 57 & 0.00 \\
\hline ENSMUSG00000030669 & 0 & 36 & 0.00 & ENSMUSG00000024975 & 436 & 1087 & 0.00 \\
\hline ENSMUSG00000020108 & 18 & 133 & 0.00 & ENSMUSG00000050071 & 0 & 10 & 0.00 \\
\hline ENSMUSG00000021388 & 16 & 120 & 0.00 & ENSMUSG00000036333 & 73 & 189 & 0.01 \\
\hline ENSMUSG00000008999 & 0 & 29 & 0.00 & ENSMUSG00000060216 & 563 & 1334 & 0.01 \\
\hline ENSMUSG00000006205 & 20 & 130 & 0.00 & ENSMUSG00000062312 & 0 & 9 & 0.01 \\
\hline ENSMUSG00000038508 & 20 & 127 & 0.00 & ENSMUSG00000026383 & 0 & 9 & 0.01 \\
\hline ENSMUSG00000040488 & 6 & 59 & 0.00 & ENSMUSG00000031681 & 241 & 555 & 0.01 \\
\hline ENSMUSG00000024598 & 0 & 24 & 0.00 & ENSMUSG00000033585 & 2 & 52 & 0.00 \\
\hline ENSMUSG00000046058 & 17 & 96 & 0.00 & ENSMUSG00000021214 & 113 & 659 & 0.00 \\
\hline ENSMUSG00000022150 & 264 & 971 & 0.00 & ENSMUSG00000006930 & 9 & 35 & 0.01 \\
\hline ENSMUSG00000042757 & 49 & 204 & 0.00 & ENSMUSG00000059146 & 2 & 981 & 0.00 \\
\hline ENSMUSG00000031074 & 0 & 20 & 0.00 & ENSMUSG00000029530 & 131 & 4774 & 0.00 \\
\hline ENSMUSG00000023047 & 0 & 20 & 0.00 & ENSMUSG00000029379 & 0 & 159 & 0.00 \\
\hline ENSMUSG00000034612 & 0 & 20 & 0.00 & ENSMUSG00000021702 & 0 & 110 & 0.00 \\
\hline ENSMUSG00000032726 & 0 & 19 & 0.00 & ENSMUSG00000031780 & 217 & 2882 & 0.00 \\
\hline
\end{tabular}


Page 18/24 


\begin{tabular}{|c|c|c|c|c|c|c|c|}
\hline Gene ID & $\begin{array}{l}\text { PDR } \\
\text { count }\end{array}$ & $\begin{array}{l}\text { SKYY_ } \\
\text { count }\end{array}$ & $\begin{array}{l}p \\
\text { value }\end{array}$ & Gene ID & $\begin{array}{l}\text { PDR } \\
\text { count }\end{array}$ & $\begin{array}{l}\text { SKYY_ } \\
\text { count }\end{array}$ & $\begin{array}{l}p \\
\text { value }\end{array}$ \\
\hline ENSMUSG00000047898 & 0 & 87 & 0.00 & ENSMUSG00000047379 & 174 & 416 & 0.01 \\
\hline ENSMUSG00000042306 & 0 & 41 & 0.00 & ENSMUSG00000031443 & 596 & 1380 & 0.01 \\
\hline ENSMUSG00000000869 & 1 & 49 & 0.00 & ENSMUSG00000009281 & 621 & 1427 & 0.01 \\
\hline ENSMUSG00000027962 & 512 & 2993 & 0.00 & ENSMUSG00000023235 & 145 & 341 & 0.01 \\
\hline ENSMUSG00000032118 & 0 & 36 & 0.00 & & & & \\
\hline ENSMUSG00000048251 & 144 & 701 & 0.00 & & & & \\
\hline ENSMUSG00000055994 & 3 & 47 & 0.00 & & & & \\
\hline ENSMUSG00000052957 & 3 & 44 & 0.00 & & & & \\
\hline ENSMUSG00000040026 & 27911 & 115085 & 0.00 & & & & \\
\hline ENSMUSG00000056427 & 0 & 25 & 0.00 & & & & \\
\hline ENSMUSG00000022015 & 0 & 24 & 0.00 & & & & \\
\hline ENSMUSG00000021508 & 24 & 128 & 0.00 & & & & \\
\hline ENSMUSG00000042190 & 205 & 820 & 0.00 & & & & \\
\hline ENSMUSG00000026235 & 0 & 22 & 0.00 & & & & \\
\hline ENSMUSG000000062380 & 74 & 308 & 0.00 & & & & \\
\hline ENSMUSG00000037868 & 30 & 140 & 0.00 & & & & \\
\hline ENSMUSG00000025959 & 134 & 517 & 0.00 & & & & \\
\hline ENSMUSG00000028126 & 56 & 230 & 0.00 & & & & \\
\hline ENSMUSG00000033542 & 0 & 19 & 0.00 & & & & \\
\hline ENSMUSG00000019122 & 31799 & 107706 & 0.00 & & & & \\
\hline ENSMUSG00000014158 & 16 & 77 & 0.00 & & & & \\
\hline ENSMUSG00000041120 & 175 & 585 & 0.00 & & & & \\
\hline ENSMUSG00000029371 & 0 & 16 & 0.00 & & & & \\
\hline ENSMUSG00000038668 & 32 & 122 & 0.00 & & & & \\
\hline ENSMUSG00000021194 & 0 & 15 & 0.00 & & & & \\
\hline ENSMUSG00000028341 & 0 & 15 & 0.00 & & & & \\
\hline ENSMUSG00000029071 & 30 & 115 & 0.00 & & & & \\
\hline ENSMUSG00000026640 & 0 & 14 & 0.00 & & & & \\
\hline ENSMUSG00000062991 & 32 & 111 & 0.00 & & & & \\
\hline ENSMUSG000000020900 & 0 & 13 & 0.00 & & & & \\
\hline ENSMUSG00000072596 & 4981 & 13817 & 0.00 & & & & \\
\hline ENSMUSG00000037613 & 14 & 59 & 0.00 & & & & \\
\hline ENSMUSG00000059714 & 877 & 2413 & 0.00 & & & & \\
\hline ENSMUSG00000055633 & 6 & 31 & 0.00 & & & & \\
\hline ENSMUSG00000031385 & 0 & 11 & 0.00 & & & & \\
\hline ENSMUSG00000042265 & 799 & 2015 & 0.00 & & & & \\
\hline ENSMUSG00000061731 & 134 & 349 & 0.00 & & & & \\
\hline ENSMUSG00000019194 & 58 & 160 & 0.00 & & & & \\
\hline ENSMUSG00000074657 & 0 & 10 & 0.00 & & & & \\
\hline ENSMUSG00000039115 & 37 & 100 & 0.01 & & & & \\
\hline
\end{tabular}


Figures

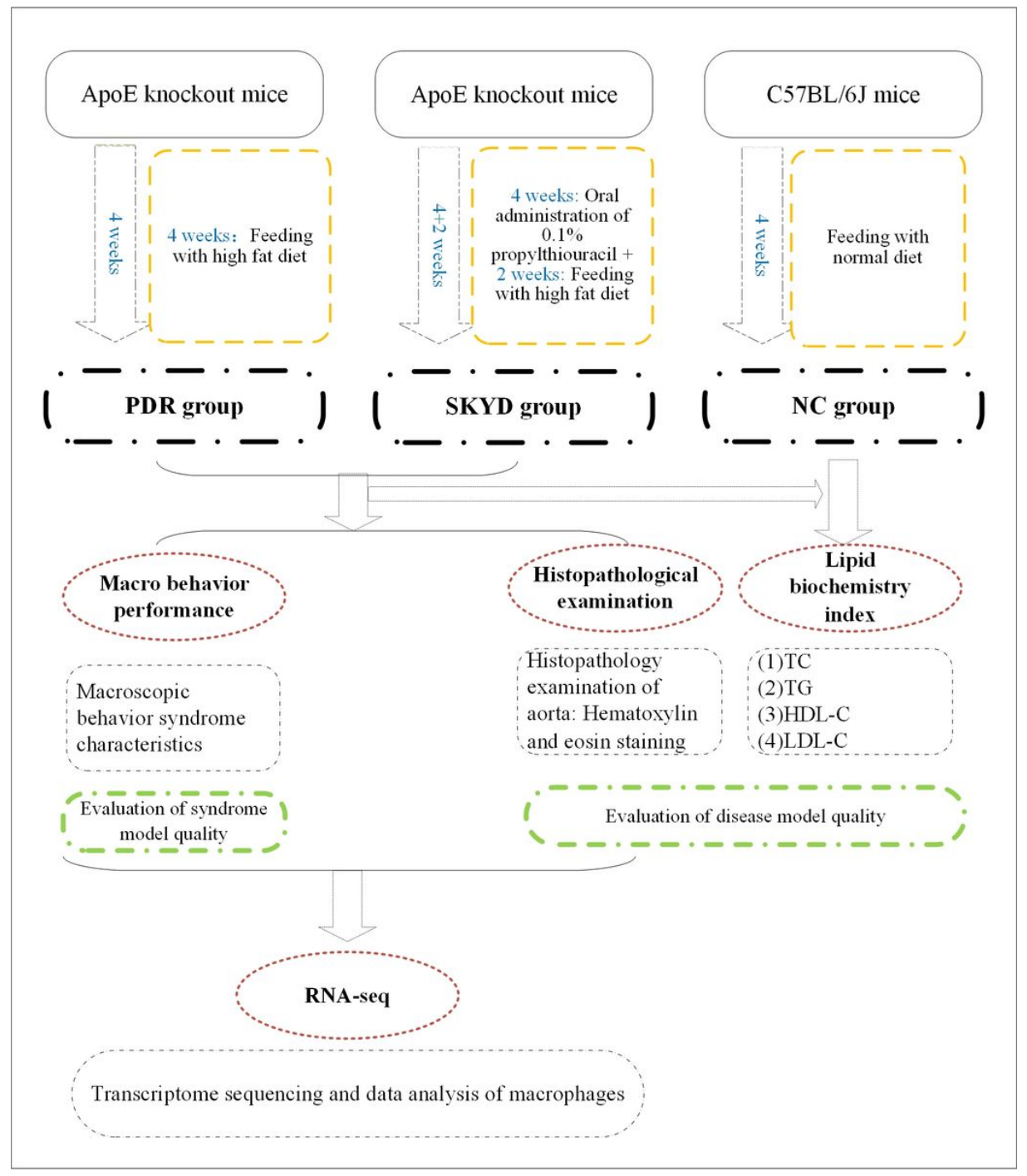

Figure 1

Flowchart of the study. 


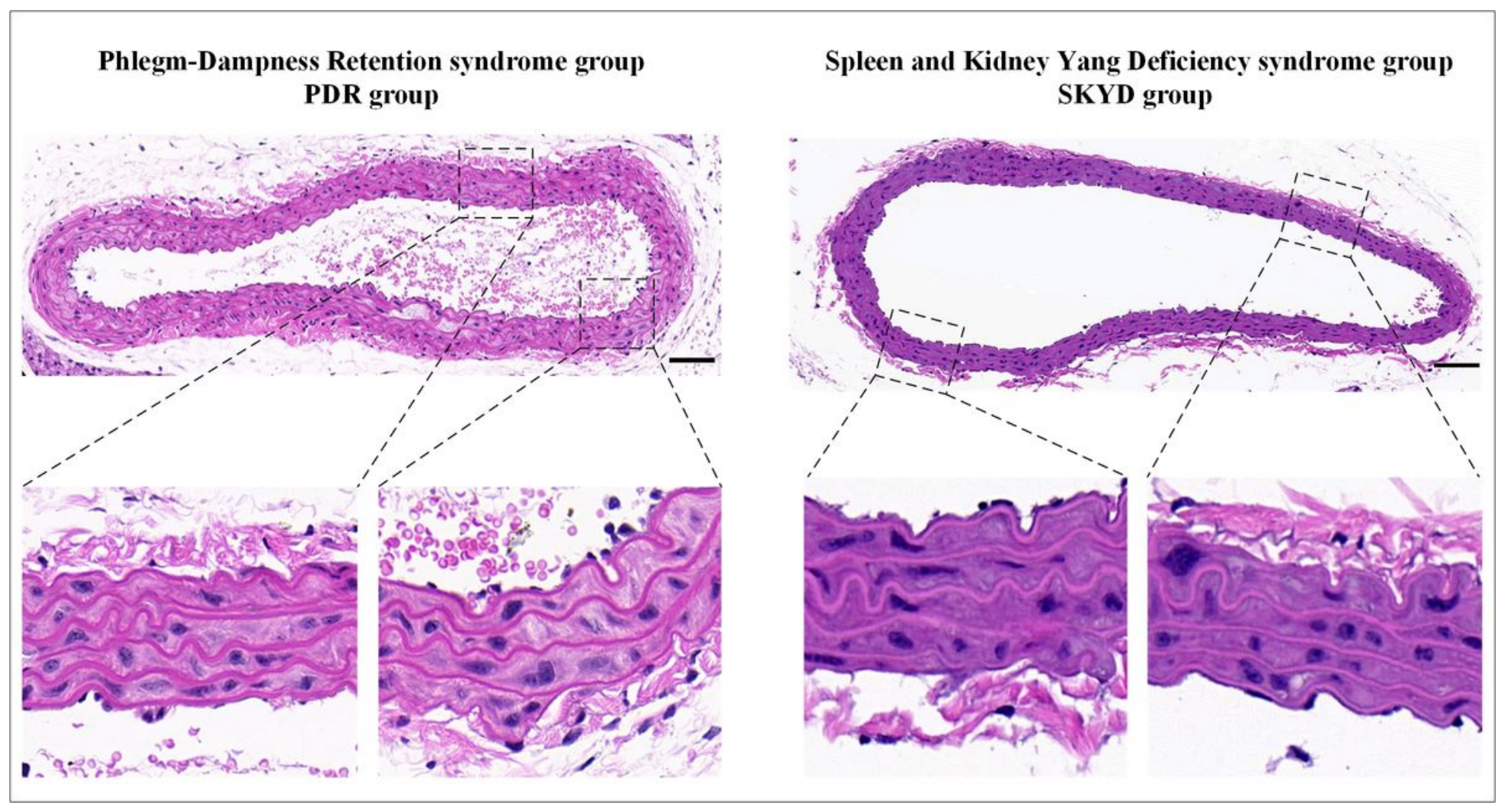

Figure 2

The aorta was stained with hematoxylin and eosin. Scale bars represent $100 \mu \mathrm{m}$. 


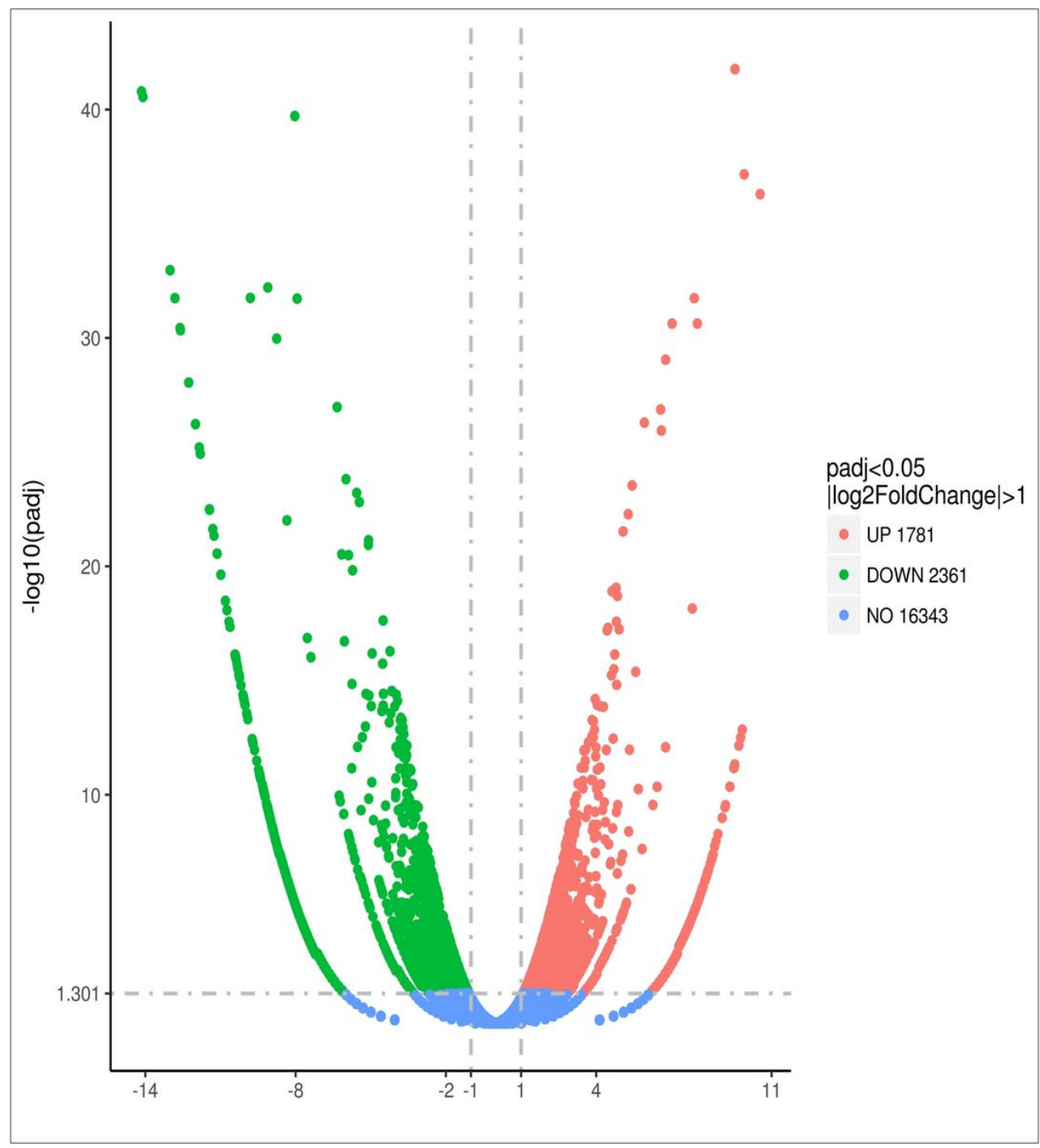

Figure 3

Quantitative comparison of gene expression levels in aortic macrophages of mice with dyslipidemia. Quantitative comparison of gene expression levels in aortic macrophages of mice with dyslipidemia: Phlegm-Dampness Retention syndrome group (PDR group) VS Kidney Yang Deficiency syndrome group (SKYD group). 


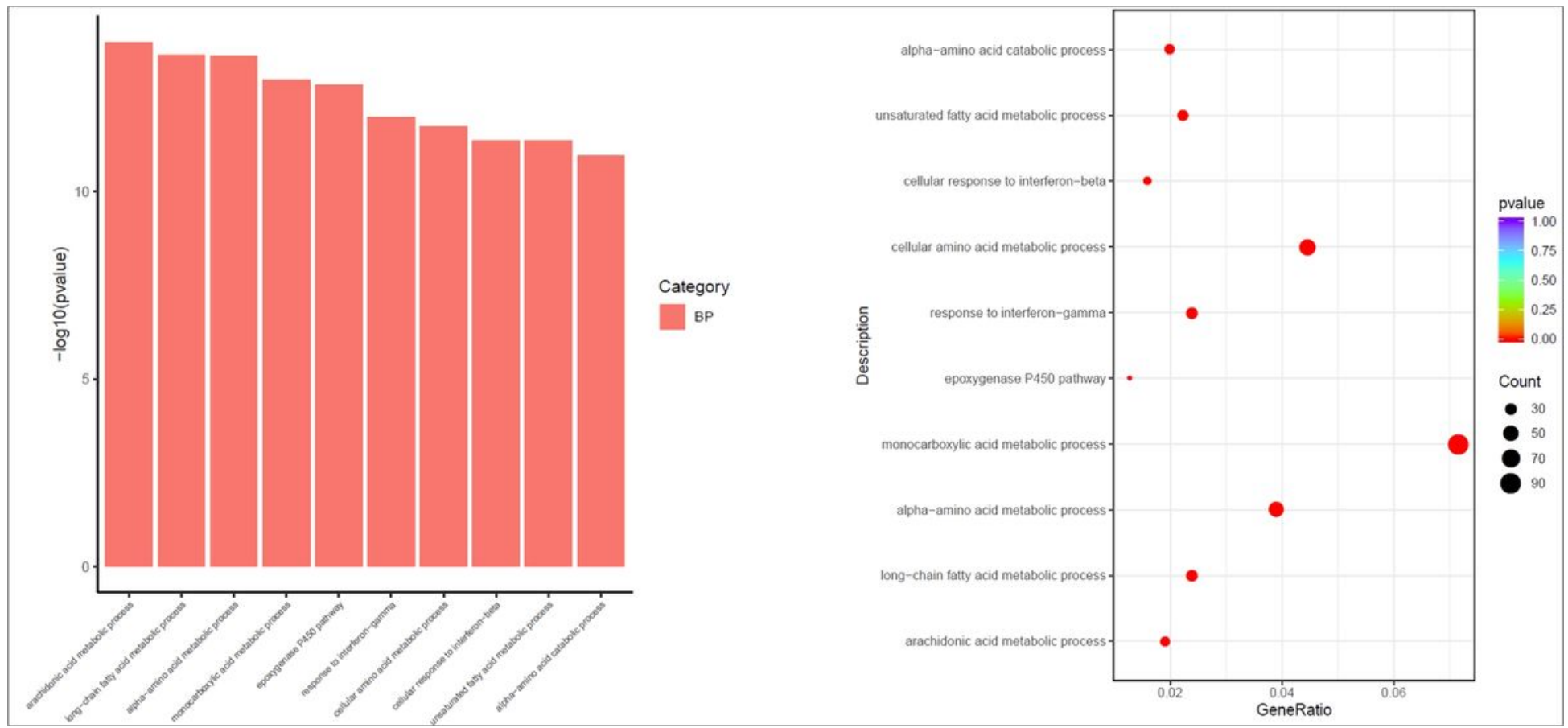

Figure 4

Different biological process of upregulation the PDR syndrome compared with the SKYD syndrome. Different biological process of upregulation in the group with Phlegm-Dampness Retention syndrome compared with the group with Kidney Yang Deficiency syndrome: Bar graph and Dot plot.

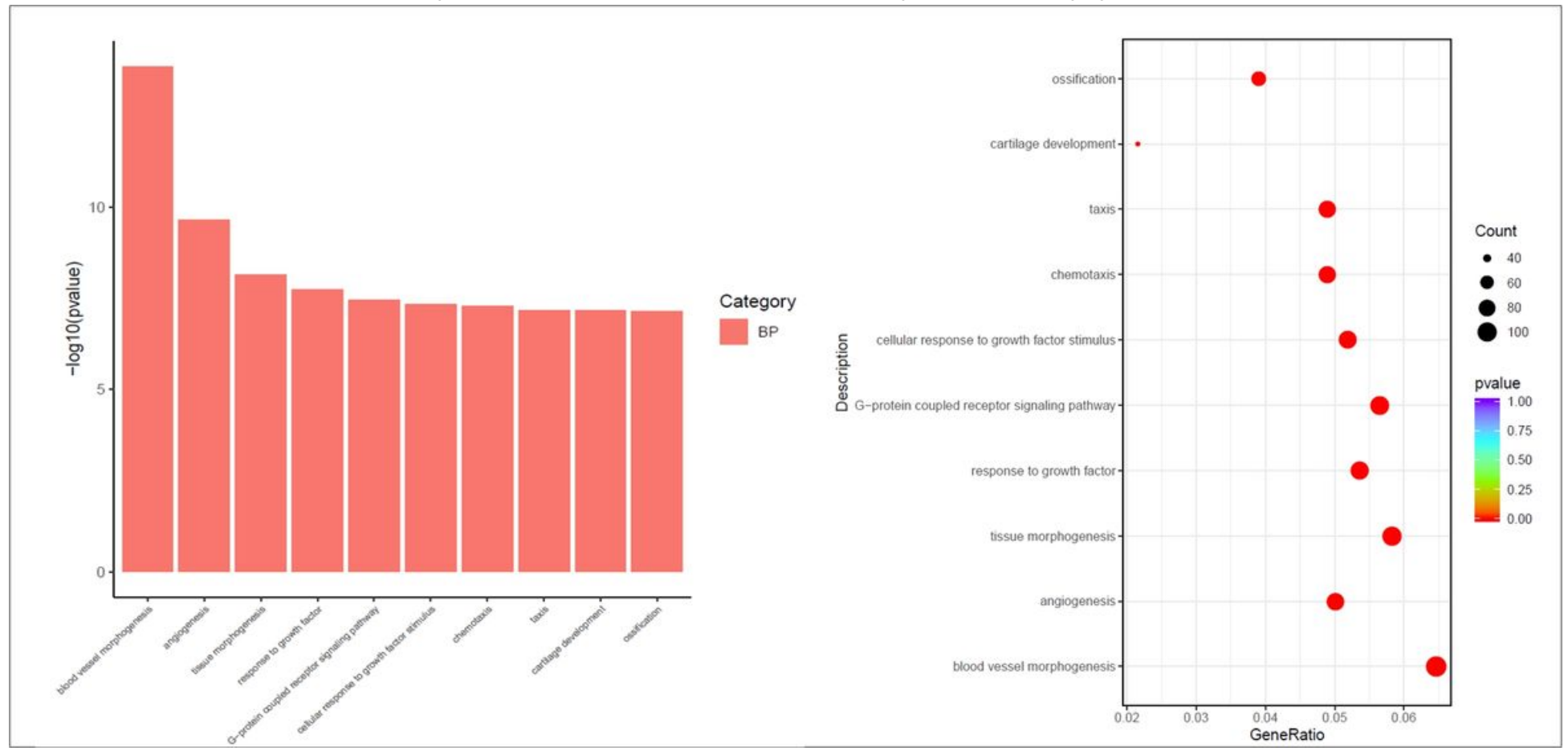

Figure 5

Different biological process of upregulation the SKYD syndrome compared with the PDR syndrome. Different biological process of upregulation in the group with Kidney Yang Deficiency syndrome compared with the group with Phlegm-Dampness Retention syndrome: Bar graph and Dot plot 


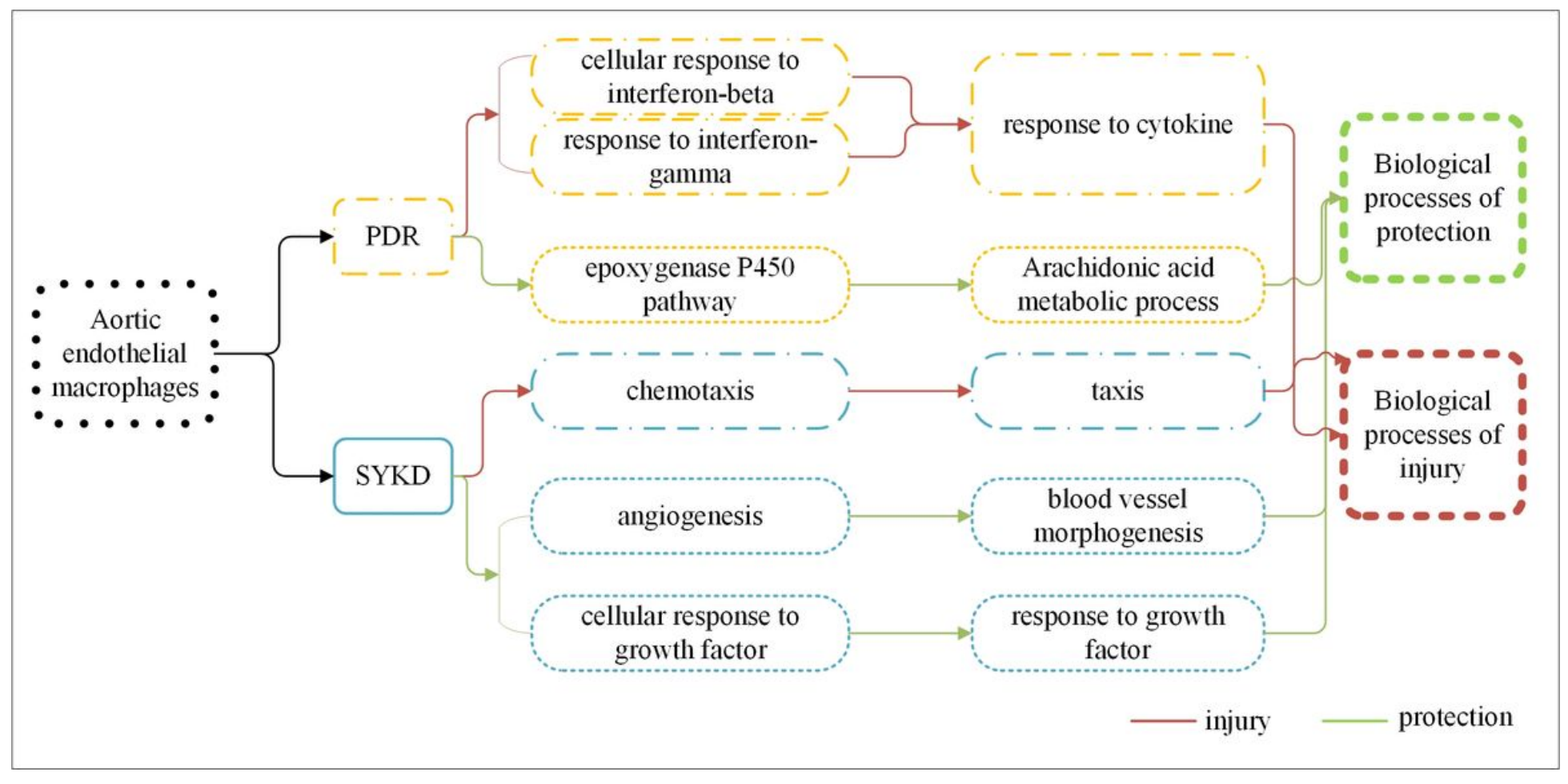

Figure 6

There are different biological processes of macrophages in dyslipidemia PDR syndrome and SKYD syndrome; There are different biological processes of macrophages in dyslipidemia Phlegm-Dampness Retention syndrome group (PDR) and Kidney Yang Deficiency syndrome group (SKYD);

\section{Supplementary Files}

This is a list of supplementary files associated with this preprint. Click to download.

- Highlights20210219.docx

- GraphicalAbstracts.jpg 